\title{
EVA London 2021 Workshops
}

\author{
Carl Hayden Smith \\ Ravensbourne University \\ London, United Kingdom \\ c.smith@rave.ac.uk
}

\author{
Daniel Buzzo \\ Creative Technology Lab \\ University of the West of England \\ Bristol, United Kingdom \\ daniel.buzzo@uwe.ac.uk
}

\author{
Eyal Gruss \\ $<3$ \\ Tel-Aviv, Israel \\ eyalgruss@gmail.com
}

\section{THE MUSEUM OF CONSCIOUSNESS WORKSHOP}

\author{
Carl Hayden Smith, Ravensbourne \\ University, London, UK
}

\subsection{Introduction}

The Museum of Consciousness is a decentralised Mixed Reality platform that allows artists to regenerate their work within a novel real-time circular system. The museum contains of a multitude of artists, mavericks and consciousness explorers who are keen to transmit their most treasured work in a way that achieves maximum impact both for themselves and for their audience.

We launched the first exhibition at Burning man in 2020. The exhibition involved 50 artists each recording a piece of audio that was designed to create an altered state within the listener. The exhibition consisted of many different types of experience including thought experiments, sound journeys, death experiments as well as sleep and dream incubations.

The core operating principles of the museum are themselves regenerative and modular. They currently consist of the following:.

\subsection{Experience stacking}

One core aim of the museum is to encourage the regeneration of the individual and collective imagination. The visitor is able to create recipes for consciousness augmentation thanks to the modular declarative structure of the museum. As they navigate through the museum the visitors are encouraged to mix and match their chosen exhibits in order to enable a process of stacking. This is intended to shift their awareness, adventure their senses and enable curious ways of seeing and being in the World.

\subsection{Declarative UX / UI}

Artists are able to regenerate their work in real-time by also being part of this stacking process. This is because the declarative $\mathrm{UX/UI}$ is designed to ensure that the ordering of experiences is always different. The basic premise is that if the experience before and the experience after changes then this will inspire regeneration and impact. Magic happens in the combinations, regenerative recipes for the augmentation of the imagination and therefore consciousness is the aim.

\subsection{Circular system}

The circular system at the heart of the museum operation consists of three intersecting parts and is designed to empower both the artists and the audience in equal measure:

i) Transmission - where the artist transmits their artwork to the audience.

ii) Experience -where the audience integrates and spends time processing the artwork.

iii) Feedback - where the audience directly communicates their thoughts and feelings back to the artist.

This circular system is designed to act as an engine for creative regeneration between the artist and the audience, maximising the impact of the interaction.

\subsection{Decentralised system}

Each artist involved 'owns' their own space in the museum. They can add, delete, work with other artists and self-organise exhibitions. They own their own data at all times. They can choose to monetise access to their experience space or not.

\subsection{Types of implementation}

The museum exists across multiple realities including the virtual, the physical and a blended 
between the two. Like mycelium, each context the museum operates in generates further contexts and this is how the museum grows.

\subsection{Types of artwork that can be housed in the museum}

Audio, visual, VR, AR, XR, sensory augmentation.

\subsection{Types of exhibition (planned)}

- Meditation techniques

- Zen koans

- Holographic storytellers

The workshop will consist of a deep dive into the operating principles of the museum and how you can make your space in the museum. Over the two hours we will organise our own exhibition together.

\section{INTRODUCTION TO GENERATIVE DRAWING}

Daniel Buzzo, University of the West of England, Bristol, UK



Figure 1: Output from a generative system.

\subsection{Introduction}

This workshop will introduce participants to the basic principles of generative computational drawing systems and give practical hands on demonstrations of coding simple generative systems for art using the popular $\mathrm{C}++$ toolkit 'openFrameworks'.

Beginning with a discussion of generative approaches to drawing from traditional media, printmaking and weaving, participants in the workshop will gain an insight into some of the long and fascinating history of generative approaches in art. Translating ideas to audio and video, from Mozart's dice music through the work of Printmaker Sol Lewitt, weaver Annie Albers and computational artist Vera Molnar to Eno's generative video and sound using computer systems.

This workshop is suitable for those with an interesting in computational and visual arts, or any familiarity with basic programming or programming principles. Demonstration code and examples will be pre-prepared enabling all participants to make their own audio or video/visual generative artwork in the course of the day or after the workshop's session.

The workshop provides an overview and grounding in the theory, history and application of generative and procedural systems in art. This shirt workshop engages participants in a discussion of theory and practical application of systems featuring exemplar material from creative practitioners from the field of music and visual arts.

Through the practice of systems of drawing translated into computational drawing, participants will be introduced to the mathematics and philosophical foundations of algorithmic and generative approaches to creation alongside practical demonstration and experimentation with systems. After joining this workshop participants will be able to select and apply a variety of generative systems techniques to differing situations in their own area of arts and design practice.

\subsection{Workshop activity outline}

The workshop will cover the following areas (flexible dependant upon delivery format)

- Introduction to generative and procedural systems and discussion of the historical context in relation to drawing and creativity

- including Mozart, Sol Lewitt, Annie Albers, Vera Molnar, John Cage, William Burroughs, Brian Eno, Golan Levin etc.

- Basic systems types and exemplar materials in written form/ paper drawings and in $\mathrm{C}++$, openFrameworks systems and exemplar/demo materials, Stochastics and Random Walks, Markov chains, L systems, Non linear composition.

- Participants write rules for pencil drawings and test them on paper // After the workshop

- Participants can re-work pencil drawing rules into computer code

- Participants can work on expanding demo code to their own area of interest. 


\section{AVATARS4ALL}

Eyal Gruss, Tel Aviv, Israel

\subsection{Introduction}

With the advance of the Coronavirus pandemic in the beginning of 2020, The majority of human social activity has been forced online to the virtual realm. Only a few months earlier, the First Order Motion Model (FOMM) (Siarohin et al 2019) was released, introducing the ability of one-shot videodriven image animation. Soon followed by (Aliev \& Iskakov 2020), a real-time environment for FOMM allowing using "Avatars for Zoom, Skype and other video-conferencing apps". Is the time ripe to claim the once promised cybernetic utopia? Could we at last shed our physical shells and be whoever we want to be in Zoom-space?

People come to the Oasis for all the things they can do, but they stay because of all the things they can be: tall, beautiful, scary, a different sex, a different species, live action, cartoon, it's all your call. (Ready Player One film, 2018)

The repository (Gruss 2020) contains a few Colab notebooks that attempt to make the technology accessible for all. Requiring only a browser and a Google account, these notebooks can be operated with one click ("run all"). However, they are also flexible tools, allowing users to use and manipulate their own selected media. The live webcam environment is based on WebSocket similar to webcam Google Colab (2020). To the author's best knowledge, it is the fastest purely online solution for live FOMM avatars, as well as one of very few realtime webcam Colab implementations. The GUI in figure 1 shows a multitude of controls for zooming, calibration, switching between avatars, generating new avatars, and various model and display parameters.

A novel fast auto-calibration mode that works in real-time, finds the best alignment between driver and avatar based on model keypoints (rather than facial landmarks). Following Avatarify (Aliev \& Iskakov 2020), which inspired this project, the user can generate new avatars based on StyleGAN "This Person Does Not Exist" website. Taking the idea further, one can also generate avatars specifically of men, women, boys, girls, e.g. Fake Face, Waifus, Fursonas and Muppets (Adler \& Gruss 2020), the latter developed especially for this project by Doron Adler, in collaboration with the author. One can also drag and drop local or web images on the GUI to upload new avatars, as inspired by FaceSwap. Other innovations include an exaggeration factor slider to lever stronger keypoint motions, an option to take your own snapshot and puppeteer it, reminiscent to Nvidia Maxine, which may help understanding the mechanism, an optional post-process step for the pipeline for offline videos, using Wav2Lip (Prajwal et al 2020) following FOMM, to fix the lip sync, and combining Wav2Lip with speaker diarization for automatic animated skit creation from audio ("Wav2Skit").

These tools were the basis for several workshops and tutorials at international festivals and conferences in 2020, including Suoja/Shelter, South Africa NAF, ADAF, Reclaim Futures, Fubar, ISEA, Technarte, EVA London, Piksel, Stuttgarter Filmwinter, Dorot-Con and MozFest (Gruss 2020) They are now being introduced in elementary and middle schools in Israel with the Pisga-Cyber excellence program. A pleasantly surprising first real-world usage of the described system.

\subsection{Broader impact and ethical implications}

This is a dangerous time. The ability to synthesize and manipulate media is improving by the day. In the quality of outcome, in the mediums, modalities and conditions dealt with, in the required compute and data resources, and in the availability and accessibility of the technology. We are in the midst of a transition period, were these facilities are still accessible mostly to the tech savvy and those of means to hire them. It may not be long, before we have ubiquitous and seamless smartphone apps that can create perfect deep fakes. However, it is the author's opinion that precisely in this interim, it is imperative to liberate and democratize the technology.

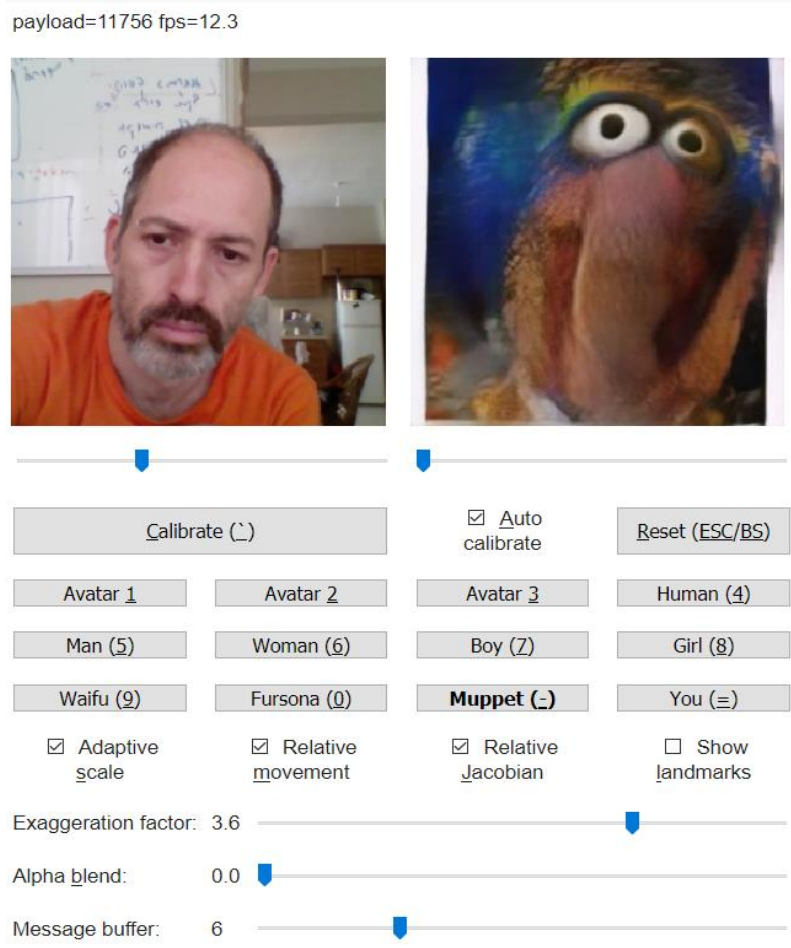

Figure 2: GUI for live webcam avatar in Colab. The author (left) is puppeteering a generated Muppet. 


\section{REFERENCES}

Adler, D and Gruss, E. (2020) This muppet does not exist. https://thismuppetdoesnotexist.com

Albers, A. (1974) On Weaving. Wesleyan University Press

Aliev, A and Iskakov, K. (2020) Avatarify. https://github.com/alievk/avatarify

Face Swap [Online interactive] https://terryky.github.io/tfjs_webgl_app/face_landm ark

Fake Face [Online interactive]. https://fakeface.rest

Generative Art, Science and Technology hard Journal (GASATHJ). http://www.gasathj.com/tikiindex.php

Gruss, E (2020) Avatars4all [Github]. https://github.com/eyaler/avatars4all

LeWitt, S. (1977, Reprinted 2019) Four Basic Kinds of Lines \& Colour. Primary Information/ Printed Matter, Inc.

Lostritto, C. (2019) Computational Drawing. Applied Research \& Design Inc.

Nvidia Maxine.

https://developer.nvidia.com/MAXINE

Pisga-Cyber. https://pisgacyber.co.il

Prusinkiewicz, P. and Lindenmayer, A. (2004) The Algorithmic Beauty of Plants Springer-Verlag, New York
Prajwal, K.R., Mukhopadhyay, R., Namboodiri, V., and Jawahar, C.V. (2020) A lip sync expert is all you need for speech to lip generation in the wild. http://bhaasha.iiit.ac.in/lipsync

Reas, C. and McWilliams, C. (2010) Form+Code in Design, Art, and Architecture. Princeton Architectural Press.

Shiffman, D. (2012) The Nature of Code: Simulating Natural Systems with Processing. Nature of Code, NY.

Siarohin, A., Lathuilière, S., Tulyakov, S., Ricci, E., and Sebe, N. (2019) First order motion model for image animation. In $\mathrm{H}$. Wallach, $\mathrm{H}$. Larochelle, A. Beygelzimer, F. d'Alché-Buc, E. Fox, and R. Garnett, (eds) Advances in Neural Information Processing Systems 32, pp. 7137-7147. Curran Associates, Inc. https://aliaksandrsiarohin.github.io/first-ordermodel-website

This Fursona Does Not Exist [Online interactive]. https://thisfursonadoesnotexist.com

This Person Does Not Exist. https://thispersondoesnotexist.com

This Waifu Does Not Exist. https://www.thiswaifudoesnotexist.net

Webcam Google Colab (2020) [Github]. https://github.com/a2kiti/webCamGoogleColab 